Belli, S. (2014). El rol de las emociones en el diálogo intercultural. Implicaciones educativas. Revista Electrónica Interuniversitaria de Formación del Profesorado, 17 (2), 109-122.

DOI: http://dx.doi.org/10.6018/reifop.17.2.197551

\title{
El rol de las emociones en el diálogo intercultural. Implicaciones educativas
}

\author{
Simone Belli \\ Universidad Carlos III de Madrid
}

\section{Resumen}

El objetivo de este trabajo es mostrar la complejidad de las situaciones comunicativas multilingües, y de qué manera las emociones se mezclan con las prácticas de los nuevos hablantes y su entorno social. En segundo lugar se quiere reflexionar sobre el impacto de las practicas lingüísticas, centrándonos en los efectos que presentan sobre las personas en términos de poder y discriminación social. Se ha analizado los datos del trabajo etnográfico en las aulas de enlace de la Comunidad de Madrid y se ha utilizado el Análisis de las Emociones en contextos interculturales. Los efectos producidos en las prácticas cotidianas a través prejuicios y construcción de lugares comunes a través el lenguaje en la relación profesorado/alumnado. En la comunicación intercultural se hacen evidentes prácticas de discriminación y de marginalización de los nuevos hablantes.

\section{Palabras clave}

Nuevo hablante; emociones negativas; malestar; análisis del discurso; interculturalidad.

\section{The role of emotions in intercultural dialogue. Educational implications}

Contacto

Simone Belli , simone.belli@uc3m.es, Despacho: 17.02.37. Edificio ORTEGA Y GASSET. Universidad Carlos III de Madrid.

Financiación recibida a través del programa postdoctoral Alianza 4 Universidades Universidad Autónoma de Madrid. 


\section{Abstract}

The aim of this work is to show the complexity of multilingual communicative situations, and how emotions are mixed with new speakers practices and their social environment. In the second place we want to reflect on the impact of linguistic practices, focusing on the effects that have on people in terms of power and social discrimination. These effects are produced through everyday practices and prejudices platitudes building how we will see in the examples. What concerns us mostly of these social practices are the linguistic attitudes how speakers usually have, and are mostly visible in intercultural communication.

\section{Key words}

New speakers; negative emotions; unrest; Discourse Analysis; Interculturality.

\section{Introducción}

Se presenta aquí una etnografía sobre multilingüismo en el aula en un programa lingüístico educativo para recién llegados de inmersión al español. El estudio ha puesto en evidencia las dificultades de hablar correctamente un nuevo idioma por parte de un hablante, lo que origina muchas veces sentimientos de enfado, enojo, frustración o miedo que se convierten en fuente de malentendidos por parte de quien lo escucha, dando lugar a prácticas alternativas o subversivas para expresar tales emociones (Belli, 2013, 2014).

En el presente artículo se tratará la relación entre la dificultad de hablar correctamente un idioma por parte de un nuevo hablante y el malestar producido en sus prácticas sociales, que se convierte en fuente de malentendidos por parte de quien lo escucha, y que muchas veces da lugar a sentimientos de enfado, enojo, frustración o miedo. Miedo a hablar una lengua que no se conoce perfectamente y miedo que se produce al viajar a un país donde no se habla la propia lengua. Este miedo a relacionarse con los demás es lo que muchas veces hace callar a los nuevos hablantes que no pueden decir lo que quieren, produciendo malestar y malentendidos en su situación de interacción.

El material analizado se compone de ocho entrevistas a cuatro alumnas, realizadas en dos semestres diferentes del mismo año escolar (diciembre y junio). El número de los participantes en cada entrevista se reduce a dos (entrevistador-entrevistado), aunque en algunos momentos aparece una tercera persona (profesora). Estas alumnas son estudiantes sin nacionalidad española que participan al programa de educación lingüística que ofrece la Comunidad de Madrid denominado Aula de enlace. El programa se basa en el aprendizaje del español como segunda lengua para estudiantes recién llegados que no conocen la lengua principal de instrucción.

Tres de los cuatros sujetos entrevistados, Suad, Fátima y Nadia, comparten casi la misma experiencia migratoria: han viajado desde Marruecos a España con su familia y viven en barrios donde la presencia de inmigrantes es elevada. El cuarto entrevistado, Omar, vive en una residencia de Madrid, y ha venido a España a la edad de 13 años solo, sin padres ni parientes. Las entrevistadoras son investigadoras pertenecientes al grupo MIRCo.

\section{Emociones en contextos multilingües}

En la literatura sobre multilingüismo se ha estudiado principalmente cómo los hablantes bilingües pueden cambiar de código para marcar una actitud afectiva: utilizar el primer 
idioma (L1) para señalar intimidad, sentimientos de pertenencia y expresar sus emociones, y el segundo idioma (L2) para marcar distancia, actitud respecto al exogrupo (Gumperz, 1982; Zentella, 1997).

En el caso especifico de las aulas multilingües, a los hablantes no les está permitido expresarse en ambos idiomas, L1 y L2, a través un intercambio de códigos. En la mayoría de los casos tienen que esforzarse por expresar sus emociones en el nuevo idioma, el español. Es por esta razón, que para esta investigación ha sido muy relevante el trabajo de Aneta Pavlenko $(2004,2008)$ sobre emociones en contextos multilingües. En el análisis de las emociones en contextos interculturales según Pavlenko (2008), las emociones en general pueden ser expresadas a través:

- Palabras emocionales (EW);

- Palabras relacionadas a emociones (ERW);

- Palabras cargadas de emociones (ELW).

Sin embargo, una crítica al trabajo de Pavlenko, que puede ser compartida con numerosos trabajos en el área de la psicolingüística y sociolingüística, se encuentra en la dificultad de encontrar estas palabras en la lengua, que, según Pavlenko, parecen localizarse de manera cristalina y bien definida. A este problema se añade una ulterior dificultad que consiste en individuar estas categorías de palabras en procesos multilingües. Por esta razón, aunque se intenta individuar este malestar social empezando por un análisis de las entrevistas según las instrucciones que nos ofrece Pavlenko, intentaremos hacer una distinción diferente a la que la autora nos propone y que aquí hemos descrito. En lugar de limitarnos a buscar estas palabras relacionadas con emociones, trabajaremos con la entera expresión emocional en vivo, es decir, no aislándola de su contexto. Esta expansión del planteamiento de Pavlenko ha demostrado ser muy satisfactorio cuando se analizan datos provenientes de entrevistas y narrativas, porque estas expresiones emocionales resultan mucho más elaboradas y complejas que los cuestionarios o encuestas empleadas para categorizar estas emociones. Las emociones en contextos interculturales resultan también mucho más complejas y elaboradas que en los contextos monolingües.

\section{Malestar social en aulas multilingưes}

Gracias a las entrevistas y a la observación participante en el aula multilingüe, y al planteamiento teórico que se ha descrito anteriormente, se ha intentado hacer el análisis de los datos contestando a las siguientes tres preguntas:

1. ¿Qué emociones aparecen en un aula multilingüe?

2. ¿Quién y cómo las expresa?

3. ¿Qué diferencia hay entre estas expresiones de emociones?

En el análisis, cuando se observan expresiones de tristeza, aburrimiento, enfado y miedo, se describirá estas emociones según lo que los hablantes expresan, sus descripciones de un mismo proceso. Aunque no se entrará en detalles semánticos, se ofrece a la lectora la posibilidad de comprender esta delicada dinámica en los contextos multilingües. Se analizará cada una de estas emociones por separado, para que al final se pueda entender en qué consiste este malestar social expresado en un aula multilingüe. 
Tristeza

- Extracto 1

1. Profesora 1: (qué triste está)

2. Laura (Investigadora grupo MIRCo): está triste

3. Profesora 1: (esta chica)

4. Laura: no te pongas triste / que no pasa nada / ya otro día lo

5. haces bien // ¿vale?

6. Profesora 2: claro / si no te ha puesto que esté mal / te ha puesto

7. solo / el tipo de hoja que tienes que utilizar

8. Fatima: pero / ¿sabe nota?

9. Profesora 1: es que ella quiere una nota

- Extracto 2

21. Profesora 1: aprovechar / ¿sabes? / porque ahora mismo ella /

22. quiere / integrarse como una más / pero yo le digo que es muy

23. difícil / que no puede / pero // no le gusta / porque di que en

24. música sacó un seis / y yo / qué bien / qué bien / un seis / y le

25. parecía poco // y me dice / otros han sacado más nota // digo /

26. hombre / otros no tienen tus dificultades / ¿sabes? / pero que a

27. veces como que no se dan cuenta de las dificultades que tienen

28. Laura: huum

29. Profesora 1: \& no son conscientes de las- / no / no sé / a mí me-

30. es algo / que tampoco termino de entender mucho

31. Laura: huumm

32. Profesora 1: ¿sabes? // entonces lo que ocurre es que mientras que

33. estén en el aula de enlace / eel- / claro / no reciben un boletín

34. normal / ss- / el boletín / digamos / se lo damos nosotros

- Extracto 3

35. Profesora: "eres de las que mejor lees (... ) pero las demás

36. (pobrecitas) leen mal, no quiero decirles porque se van a poner

37. muy tristes."

Con estos tres extractos se observa una de las principales causas de este malestar en el aula: las alumnas del aula multilingüe no son iguales que las que están en las aulas "normales". Tienen dificultades que no le permiten ser consideradas alumnas "normales" según las profesoras. La obsesión de la alumna Fátima, por ejemplo, en saber la nota que la profesora le va a poner, es un claro síntoma de que ella es consciente de su condición (I.8). El seis en Música que la profesora le ha dado no es el mismo seis del resto de las compañeras, nunca es considerada estudiante legítima. Esta es la razón por la que en estos 
dos extractos aparece la palabra tristeza. La profesora y la investigadora perciben y expresan esta emoción en relación con la alumna, aunque ella no la expresa directamente. Se introduce en la entrevista a través de su inseguridad y su insistencia por saber la nota, síntomas de su malestar como estudiante, por no ser considerada "legítima". Pertenece a otro grupo, no al de los hablantes nativos y estudiantes "legítimos", sino a un endogrupo de alumnos de un aula de enlace. En las líneas 33 y 34, la profesora explica que existen dos grupos diferentes, con dos evaluaciones diferentes y dos boletines diferentes: un boletín normal y otro boletín que "digamos / se lo damos nosotros".

Este es el motivo del por qué Fátima insiste en saber la nota, y es también la causa su tristeza (líneas 1- 9). Si utilizamos la categorización de Pavlenko (2008) de las emociones, podemos identificar como palabra emocional (EW) la palabra triste (línea 1, 2, 4), que aparece como una descripción de la alumna, aunque esta EW la introducen la profesora y la investigadora en la entrevista, no directamente la alumna. Es por este motivo por el que, en lugar de hablar de palabras cargadas de emociones (ELW), se quiere tratar con frases cargadas de emociones, como por ejemplo en la línea 8 ("Pero, ¿sabe nota?), enunciado que está cargado de tristeza por no entender por qué no tiene y no aparece nota, o simplemente por qué le califican con una nota según un baremo diferente.

En el segundo extracto, al aparecer términos como "difícil”, "dificultades", y frases como "no reciben un boletín normal", se produce una iteración de esta tristeza. Se trata de palabras y frases relacionadas con emociones. Esta tristeza es una constante en el aula de enlace. El Extracto 3 hace una comparación entre varias alumnas, y aparece una EW (I.37), que también es una frase porque introduce una acción, 'se van a poner muy tristes'. Más que el miedo, las alumnas exteriorizan su tristeza, síntoma de los malentendidos que se producen en estas aulas.

\section{- Extracto 4}

38. Fatima: este también es el mismo /este es el mismo/ es el mismo

39. Laura: huum / no sé aquí // no te pongas triste / que no pasa nada

((dos líneas omitidas))

42. Fatima: ¿este? / este-

43. Laura: \& los ejercicios / si

44. Fatima: ¿todos? / ¿todo esto tenemos que dibujar aquí? / no en este

45. / ¿sabes? / esto también

45. tenemos que dibujar aquí / creo que estos / pero por qué lo

\section{6. escribo aquí / ¿por qué aquí?}

Muchas situaciones de incomprensión entre alumnado/profesorado dan lugar a sentimientos de tristeza, EW de un proceso emocional (no te pongas triste, línea 39), o de una frase cargada de emoción, frustración (pero por qué lo escribo aquí, ¿por qué aquí?). El extracto 4 ejemplifica cómo la comunicación entre profesor y alumno no siempre fluye con facilidad, muchas veces se produce cierto malestar o surgen pequeños malentendidos 0 conflictos. Emerge frustración en el relato de Fátima, causada por el malentendido de lo que la profesora quiere; la repetición a sí misma del por qué hay que hacer la práctica de aquella determinada manera. Esta frustración, como se vió en los extractos anteriores, genera tristeza en Fátima (incluso la propia entrevistadora la percibe). La frustración y la 
tristeza son emociones negativas que no permiten el proceso de aprendizaje, ni de acceso a la lengua.

Pavlenko (2008) hace una distinción entre diferentes tipos de expresión de la frustración en inglés y en griego. En la construcción de un enunciado, la frustración en estos dos idiomas es bastante diferente, aunque el efecto resulta ser el mismo. El termino frustración contiene en griego una serie de estados emocionales como incomodidad, opresión y, sobre todo, tristeza (stenahoria). También Panayiotou (2004a) presenta una variedad de contextos multilingües en que esta emoción negativa es utilizada. Estas diferentes maneras de expresar emociones negativas como la tristeza tienen que ver con la cortesía en las distintas culturas, lo que también podría estar sucediendo en el extracto que se acaba de analizar.

\section{Aburrimiento}

La segunda emoción que constituye este malestar social, y que se ha encontrado en diferentes entrevistas, es la del aburrimiento. Esta emoción es la causa de muchos de los problemas relacionados con el aprendizaje en el aula multilingüe. El aburrimiento es una emoción negativa común en casi todos los sujetos entrevistados:

- Extracto 5

47. Entrevistadora: ¿te aburres en clase?

48. Fatima: algunas veces

49. Entrevistadora: ¿con quiénes te aburres? // ¿con qué

50. profesores te aburres más o cuáles te gusta más?

51. Fatima: no sé / todos

- Extracto 6

66. Entrevistadora: ah // o sea que sí que aprendes en el

67. aula de enlace pero te aburres // \{Riendo\} es que no lo

68. entiendo muy bien // iy en la otra clase [aprendes?]

69. Omar: [me] aburro en esta clase / no sé por qué

70. / o sea que tampoco sé por qué / ¿sabes?

71. Entrevistadora: ah

72. Omar: me quedo ahí dos horas / con los mismos profesores

74. Esther: $y$ en la otra / claro / cambias de profe cada-

75. / cada cincuenta minutos / ¿no? // claro

En el análisis se ha observado cómo en la entrevista a Fátima difícilmente se encuentran frases en primera persona. Se podría pensar que oculta sus emociones y que las expresa utilizando formas verbales típicas para mostrar su malestar, frustración, impotencia (Belli, 2013, 2014). En el extracto 5, la emoción del aburrimiento viene expresada de manera indirecta por el sujeto entrevistado, su expresión es siempre introducida por la entrevistadora, pero el extracto 6 es diferente: Omar expresa de manera directa su aburrimiento, usando una palabra emocional, "me aburro". Aquí se puede notar una diferencia entre Omar y Fátima en la expresión verbal de sus emociones. El yo que se 
expresa en las narrativas de Omar, corresponde al de un sujeto legítimo y hablante español, un caso totalmente diferente al de Fátima.

Aunque las causas de este aburrimiento de Omar no están bien definidas porque ni el propio entrevistado sabe expresar los motivos (duración de la clase, profesor, clase en general), es interesante ver cómo hace una diferencia entre dos espacios definidos (69-72), en que él se posiciona como hablante legítimo (Harré, 1989, Belli, 2014). El espacio del aula de enlace, aunque resulta más provechoso para mejorar su nivel de español, posiciona el sujeto en un estado de aburrimiento. No obstante, esto deriva en una situación confusa, que tampoco la entrevistadora entiende (74-75). Esta dinámica, según Pavlenko (2008), proviene de la dificultad del sujeto de expresar su emoción de aburrimiento en el segundo idioma (L2). El entrevistado reconoce la existencia de esta EW, pero le cuesta argumentar por qué razón se produce esta emoción en el caso específico del aula de enlace. Esto podría derivar en una división causada por las diferentes maneras de expresar este malestar, este aburrimiento, en los dos idiomas de Omar. Sería interesante haber podido cambiar al idioma L1 para ver si el sujeto entrevistado hubiera podido expresar mejor este aburrimiento. En el extracto 6 este análisis hubiera sido posible porque el sujeto ha tratado el tema, en el extracto 5 este tipo de análisis no se plantea porque en la L2 el aburrimiento no es expresado directamente por la entrevistada.

Enfado

\section{- Extracto 7}

\section{Suad: no // es que algunas veces me enfado}

77. porque / uf / cada minuto y minuto un ejercicio

78. muy difícil que empezar // al principio tienes

\section{9. que coger el diccionario / buscar la pregunta /}

80. pero ahora no / no parece como al principio //

81. Entrevistadora: $\mathrm{mm}$

82. Suad: al principio me aburro y es que no sé qué es

La tercera alumna entrevistada, Suad, reproduce la emoción del enfado cuando tiene que realizar la acción de buscar una palabra en el diccionario. Esta emoción del enfado la contrasta con el aburrimiento que este acto implica (1.82). Son emociones que se contrastan, pero que nos indican el malestar que estas acciones comportan para la entrevistada: son las que componen su malestar, producido por mejorar su L2, una performance emocional (Belli, Gil, 2011) que interacciona y actúa cada vez que se produce esta frustración de abrir el diccionario y buscar la palabra del ejercicio. Estas prácticas son muy típicas en el proceso de aprendizaje de un idioma, un proceso difícil, fatigoso y complejo que requiere una buena mezcla de habilidad y motivación. Para los nuevos hablantes en las aulas de enlace se añade también una relación de poder desequilibrada y unidireccional entre profesor-alumno, con la consecuencia de que aprender un nuevo idioma puede resultar una práctica agotadora y humillante para estas alumnas.

\section{- Extracto 8}

83. Omar: no sé qué voy a aprender / ¿sabes? / pero

84. todavía soy muy pequeño // ¿sabes? // tengo catorce

85. años / todavía no sé lo que está pasando en la vida // 
86. cuando tenga dieciséis ya seré un poco mayor / ¿sabes? //

87. Entrevistadora: y algún rato os lo pasáis bien entonces ahí

88. Omar: claro // y algún rato lo pasamos fatal también //

89. Entrevistadora: ¿sí? // ¿por qué? //

90. Omar: pos castigao / castigao un- / un mes / en la

91. habitación sin salir // te suben la comida y tú en

92. la habitación //

93. Entrevistadora: ¿y tú has estado un mes así? //

94. ¿cuándo te echaron de aquí? // pero ibas a algún

95. sitio por la mañana / ¿no? // ¿no? // ¿todo el tiempo \&

96. Omar: toda la mañana estudiando // en la habitación

97. // y toda la tarde en la habitación

98. Entrevistadora: ¿un mes / así? // ¿y no salías nada /

99. nada / nada?

100. Omar: ni salir a la calle / ni jugar al fútbol

101. / no bajar al patio ni nada

102. Entrevistadora: pues si con la \&

103. Omar: ni bajar a comer

104. Entrevistadora: con la energía que tú tienes un / un / un \&

105. Esther: te volverías loco dentro de la habitación / ¿no?

106. Entrevistadora: ¿y eso fue porque te echaron de aquí? //

107. Omar: (sí)

En este extracto se puede asistir a una toma de posición por parte del entrevistado, que, contrastando la intervención de la entrevistadora (87), cuenta su devastadora experiencia personal en la residencia. Una experiencia pasada que incide en el presente $y$, seguramente, en su futuro. Lo que el sujeto relata tiene que ver más con un anécdota, porque evoca una experiencia pasada, la de su situación en la residencia, construyendo su malestar a partir de la expresión 'y algún rato lo pasamos fatal' (88). En esta narrativa también resulta importante el papel de la entrevistadora como co-constructora del relato emocional $(93,98,99,104)$.

Se pueden encontrar en este último extracto palabras relacionadas con emociones (ERW), descripciones relacionadas con este enfado sin nombrar la emoción en concreto. 'Lo pasamos fatal', 'sin salir a la calle, ni jugar a futbol', 'castigao', son palabras que se relacionan con este enfado que Omar intenta expresar. Una experiencia destructora y negativa para el protagonista contada a través su segundo idioma, haciendo emerger su malestar respecto a la presente situación. Indirectamente expresa este enfado, pero esta emoción tan negativa es causada por esta situación extrema de aislamiento en su habitación. 
Miedo

- Extracto 9

108. Fatima: ellos hacen sus ejercicios y yo sola

109. Laura: tú estás solas aparte // te atiende a ti sola

110. Profesora1: claro / sí / porque no puede seguir el ritmo

111. [de la clase]

112. Laura: [pero vamos] / que no la vais a incluir al final

113. en un grupo de diversificación completamente [este año]

114. Profesora1: [mm / no] / la idea es / que el año que

115. viene sí / entonces / que este año asista a esas clases

116. para irse poniendo un poco las pilas

La última emoción encontrada en el análisis es el miedo, una de las seis emociones primarias según Wetherell (2012). En el extracto 9, se puede observar un ejemplo de construcción del miedo en la relación entre profesor/alumna. El ritmo (110) es lo que hace de Fátima una alumna diferente a las demás, una condición que no le permitirá sentirse una estudiante legítima. En la observación en el aula, se ha visto a Fátima como un sujeto apartado, aislado y triste, por esta razón quiere pasar al otro grupo, integrándose con las demás. La profesora indirectamente introduce en la entrevistada una situación de miedo: 'porque no puede seguir el ritmo', 'para irse poniendo un poco las pilas'. Son palabras que introducen una emoción en particular en la relación profesora-alumna: el miedo a no poder hacer lo que hacen las demás compañeras, reproduciendo dinámicas de intimidación para asustar la alumna.

\section{- Extracto 10}

120. Prof 1: [¿sabes?] / ellas quieren ir a muchas asignaturas

121. / y Yu no quiere ir / Yu quiere / aprender español aquí /

122. y luego ir a clase / porque como es muy exigente

123. consigo misma / muy / perfeccionista / claro / a ella esas

124. dificultades que tiene de no hablar el idioma / pues le

125. cuesta mucho / no / digamos que / le cuesta asumir tu- /

126. sus limitaciones / sus errores / como / a todas // y

127. ella / pues eso / preferiría estar aquí siempre / entonces

128. hay que estar empujándola / porque no quiere ir a clase //

129. no quiere ir a gimnasia / no quiere ir a

130. Laura: \& huum

131. Prof1: \& / y / yo hablé para que hubiera ido a informática

132. / y no ha querido ir / y no va / bueno / incluso es que se nos

133. ha puesto a llorar un par de veces / como muy agobiada / ¿no? / 
Si en el extracto 9 se producía una situación de miedo construyendo una narrativa sobre los diferentes ritmos entre alumnos y la dificultad de conseguir un ritmo adecuado por parte de la profesora, en el extracto 10 asistimos a un caso bien diferente. Emergen palabras asociadas a emociones negativas como 'llorar' y 'agobiada', que construyen a través otras características el miedo que la alumna siente. En la descripción que la profesora hace de Yu, podemos identificar este miedo. Acciones como 'Se ha puesto a llorar un par de veces', resultan ser una actividad que se relaciona con una emoción bien definida, que en este caso es el miedo. La profesora argumenta y motiva en su entrevista la situación de Yu partiendo siempre sobre de la base del aprendizaje de L2 y de las cualidades y carencias de su alumnado respecto a L2. Por este motivo, se produce un clima de malestar social en el aula, con performances de este tipo: un llanto social, compartido de diferentes maneras entre alumnado, que expresa este miedo.

- Extracto 11

135. Fatima: mañana / tenemos examen

136. Laura: \& pero / ¿te lo sabes?

137. Fatima: no entiendo nada de su examen

138. Laura: humm // ¿qué más tienes / el jueves? [música]

139. Fatima: voy a sacar un cero

Una situación de este tipo, de malentendido (137) genera también impotencia respecto a la institución, y confusión respecto a las tareas que hay que hacer. Estas expresiones $(137,139)$ resultan ser frases relacionadas con la emoción (ERS) de tener miedo. Se puede observar una manera indirecta de expresar este miedo muy parecida en el siguiente extracto de Nadia:

- Extracto 12

140. Nadia: yo quiero un día hablar a ella / quiero

141. hablar contigo y dice / la última / y luego ella te

142. va / y yo (())

143. Laura: no has hablado con ella

144. Nadia: no

((3 líneas omitidas))

148. Nadia: y luego / pregunto al profesor / pero el profesor

149. no puede hacer todo conmigo / solo yo / por eso-

Nadia explica un intento de hablarle a la profesora que se convierte en un fracaso por ella, un relato que expresa cómo la falta de comunicación es la principal responsable de estas emociones negativas. Un proceso de incomunicación que reproduce emociones muy nocivas que no permiten crear un contexto amigable y de confianza donde el aprendizaje sea posible y no una mera imposición. Una imposición que se entiende como relación de poder entre profesor y alumno, unidireccional, donde el diálogo muchas veces no es posible. Una subjetivación del poder social y educativo (Hemmings, 2005); una situación que causa un conflicto, que se enfoca no en la imposición de la lengua, sino en la indiferencia y marginalización de los problemas que la alumna tiene en su proceso de aprendizaje. La alumna en el extracto 12 intenta explicar a la entrevistadora, pero también a sí misma, lo que está pasando, y ella misma comparte la idea equivocada de que es normal 
que sucedan estas situaciones en el aula, aislándose porque el profesor no puede hacer todo con ella y favoreciendo la inclusión de prácticas nocivas en el proceso de aprendizaje en el aula de enlace.

\section{- Extracto 13}

150. Laura: pero bueno / no te preocupes / si el próximo

151. día se lo preguntas o- / y le dices por- / ¿qué tengo

152. que hacer? / es que no he entendido / y que te lo explique

153. // ¿y por qué no tengo nota? / pregúntale todas tus dudas

La entrevistadora le aconseja a Fátima preguntar lo que no entiende, no tener miedo al tutor, compartiendo la opinión de la profesora sobre el tutor: que es majo y afable. La percepción del miedo es una emoción que muchas veces se "encuentra" en el aula. Estos miedos son los que después se persiguen en las vidas de adolescentes y de adultos, condicionando su socialización, y que constituyen sus identidades personales y sociales (Broncano, 2013). Esta sensación de tener un déficit, de no tener bastante nivel para seguir con lo que se quiere hacer en los estudios, puede causar un sufrimiento personal más allá de la vida adolescente.

\section{Cuando el malestar social es síntoma de prejuicios}

La forma en que se gestionan las lenguas revela un acercamiento que responde a los valores y prejuicios de la comunidad de acogida. Estos prejuicios compartidos entre algunos profesores de las Aulas de Enlace nos indica la emergencia del problema. En estas aulas, justamente por estas razones, la mayoría de las veces se impone un orden lingüístico, un orden que los nuevos hablantes tienen que respetar. A causa de esto, no se permite que las lenguas de la inmigración se integren, porque sólo parece estar permitido un espacio donde se impone la lengua local y, consecuentemente, eso afecta a las relaciones sociales. Si las profesoras clasifican las alumnas como estudiantes que tienen un bajo nivel, ellas actuarán como alumnas de bajo nivel. Tanto en el aula como en la sociedad, entran en juego diferentes dinámicas de categorización negativa y de prejuicio. Las profesoras, como la sociedad en general, en sus prácticas cotidianas tienden a hacer emerger estas visiones estereotipadas de la sociedad:

\section{- Extracto 14}

154. Prof: Porque claro no es lo mismo que tu lengua materna sea el

155. árabe, que tu lengua materna sea el portugués, evidentemente. Las

156. que tienen como lengua materna el árabe, tienen muchísimas más

157. dificultades que no un portugués.

\section{- Extracto 15}

158. Prof1: \& claro / entonces lo que pasa es que hay veces / que yo

159. qué sé / una raíz cuadrada / cómo explicas lo que es una raíz

160. cuadrada si no les enseñas a hacer raíces cuadradas / oo- / y- /

161. y bueno / y luego además por ayudarles / porque / como realmente

162. / tienen esas carencias desde su escuela de origen 
Si en el primer extracto se observa como actúan los prejuicios lingüísticos, en el segundo se asiste a un ejemplo de cómo aparecen prejuicios en los contextos educativos. Lo que se está haciendo en los dos ejemplos es desnaturalizar el conocimiento naturalizado que el nuevo hablante y sus compañeras, que están en la misma situación, tienen. Ni las lenguas tienen el mismo valor simbólico-económico y social, ni tampoco la educación, como se observa en estos ejemplos. No todos los sistemas educativos alcanzan el mismo valor (162), favoreciendo unas dinámicas que construyen determinadas lenguas hegemónicas y sistemas educativos distintos. Comprender qué es una raíz cuadrada (159) significa acceder a un capital simbólico y, de ahí, todas las dificultades que se generan para acceder a ese conocimiento.

En muchos de los casos observados en el aula multilingüe, los nuevos hablantes recurren a reprimir su L1, porque el uso de otro idioma que no es el español es marginalizado o excluido de las interacciones formales. Entonces, en el caso de que lo hagan, pueden producir emociones equivocadas, o que puedan ser percibidas de manera diferente para los que están en su entorno, causando más impacto en los que los rodean, cuando en realidad querían producir otro efecto al expresarse. La carga discursiva wittgensteiniana que tienen algunas palabras emocionales no es semejante en cualquier idioma. El decir "Tengo miedo" en mandarín, en ruso o en inglés, tiene una fuerza de impacto menor o mayor en el contexto en que se produzca dependiendo siempre de quién lo dice, cuándo lo dice y naturalmente, en qué lengua el nuevo hablante lo expresa, si en la nueva o en la no tan nueva.

\section{Conclusiones}

Para entender contextos de comunidades multilingüísticas y multiculturales, donde los niños nuevos hablantes tienen que negociar la comunidad de pertenencia (Brubaker y Cooper, 2000) en relación con los múltiples niveles de vigilancia y de prácticas para fortalecer la relación entre endogrupo y exogrupo por parte de miembros de la sociedad de pertenencia y de acogida, hay que tener en cuenta todos los factores que se han visto a lo largo de este artículo.

En el estudio realizado, se ha podido entender que hay dos maneras diferentes de expresar emociones en el aula. La mayoría de las veces estas emociones se pueden categorizar como negativas (frustración, enfado, etc.), claro síntoma de un malestar social. Los alumnos, dependiendo de su grado de subjetivación, las expresan explícitamente o las ocultan. Exteriorizar el aburrimiento o el enfado son prácticas sociales que están estrictamente relacionadas con los procesos identitarios del sujeto. A partir de los datos que proporcionan las etnografías, se ha podido comprender cómo los sujetos expresan su malestar con un lenguaje verbal y no verbal a veces muy explícito (un portazo, un insulto), y a veces ocultado o expresado de una manera alternativa o subversiva (hablar en otro idioma para que la profesora no entienda, escuchar música con los cascos). Se puede afirmar que existe una relación entre las emociones y los mecanismos subjetivadores generadores de identidad (Belli, 2013, 2014).

En segundo lugar, ha resultado evidente en el análisis de la relación entre la imposibilidad de hablar correctamente un idioma por parte de un nuevo hablante y el malestar producido en sus prácticas sociales, que se convierten en fuente de incomprensión por parte de quien lo escucha, y que muchas veces da lugar a sentimientos de enfado, enojo, frustración o miedo. Dado que la competencia es un fenómeno social, ya que el que alguien sea considerado competente depende de los valores sociales y de las normas sociolingüísticas, 
el malestar social detectado aparece producido, no solamente por esta dificultad de hablar con fluidez y de adecuarse al contexto, sino también por prácticas lingüísticas que a menudo enmascaran relaciones de poder bien definidas y compartidas en la sociedad actual de acogida, que a veces ejerce prácticas racistas y de discriminación social. Prácticas habituales y situaciones frecuentes, tanto en las instituciones como fuera de ellas, que generan relaciones de poder entre la sociedad de acogida y los nuevos hablantes. Se manifiestan situaciones de discriminación y de marginalización, lingüística y no lingüística, pero siempre acompañadas por una fuerte carga emocional. Y viceversa, en casos de inclusión social entre nuevos hablantes y sociedad de acogida se producen emociones positivas. Una sonrisa, una mirada cómplice.

\section{Bibliografía}

Belli, S. (2013). Voces de nuevos hablantes, voces de nuevas subjetividades. Emociones y actitudes lingüísticas en el giro poscolonial. Aposta, 56 (1), 1-27.

Belli, S. (2014). Espacios emocionales subversivos y multilingües. El cuento árabe de la niña triste. Papeles del CEIC. 101, 1-25.

Belli, S., Gil Juárez, A. (2011). Consumiendo emociones tecnológicas: la performance del "orgasmo" tecnológico. Sociología y tecnociencia: Revista digital de sociología del sistema tecnocientífico, 2 (1), 33-55.

Broncano, F. (2013). Sujetos en la niebla. Madrid: Herder.

Brubaker, R., Cooper, F. (2000). Beyond identity. Theory and society, 29 (1), 1-47.

Harré, R. (1989). Language and the science of psychology. Journal of Social Behavior \& Personality, 45, 93-129.

Hemmings, C. (2005). Telling feminist stories. Feminist Theory, 6 (2), 115-139.

Panayiotou, A. (2004a). Bilingual emotions: The untranslatable self. Estudios de Sociolinguıstica, 5, (1), 1-19.

Pavlenko, A. (2004). Negotiation of identities in multilingual contexts. New York: Channel View Books.

Pavlenko, A. (2008). Emotion and emotion-laden words in the bilingual lexicon. Bilingualism language and cognition, 11 (2), 147-173.

Thobani, S. (2007). Exalted Subjects: Studies in the Making of Race and Nation in Canada. Toronto: University of Toronto Press.

Wetherell, M. (2012), Affect and emotion: A new social science understanding, London: Sage Publications Limited.

Wilce, J. (2009a). Society, Language, History and Religion: a Perspective on Bangla from Linguistic Anthropology. The Sociology of Language and Religion, 126, 34-68.

Williams, R. (1977). Marxism and literature. Oxford: Oxford Univ. Press.

Zentella, A. (1977). Code-switching and interactions among Puerto Rican Children, Working Papers in Sociolinguistics, 50, 1-30. 


\section{Autores}

Simone Belli. Investigador Postdoctoral en la Universidad Carlos III de Madrid. Doctor en Psicología Social por la Universidad Autónoma de Barcelona, mención Doctor Europeo con la tesis 'Emociones y lenguaje'. Ha realizado estancias de investigación predoctoral en diferentes universidades extranjeras: Georgetown University, University of Manchester y Manchester Metropolitan University. Ha sido profesor visitante en la University of California, San Diego y en la Universidad Michoacana de San Nicolás de Hidalgo, Morelia. Fue investigador postdoctoral en la San Diego State University y en la Universidad Autónoma de Madrid. Ha sido profesor invitado en la Università di Bergamo (Italia) y en la University of Helsinki (Finlandia) como profesor de la Escuela de Verano en el curso "Emotions and Interculturality". Su investigación consiste en entender por qué las emociones tienen una fuerte relación con el lenguaje y cómo es posible expresar estas emociones en contextos interculturales.

\section{Agradecimientos}

El autor agradece a la Universidad Autónoma de Madrid la financiación recibida a través del programa postdoctoral Alianza 4 Universidades y a todo el grupo de investigación MIRCo para poder tener acceso al material etnográficos y a los datos de la investigación complete. De manera especial quiere dar las gracias a las cuatro investigadoras que realizaron el trabajo etnográfico: Luisa Martín, Esther Alcalá, laura Mijares y Miguel Pérez. También agradecer a la transcriptora de las entrevistas, Miriam Jiménez Bernal. El autor se ha incorporado a la investigación en la fase de análisis de las entrevistas, sin participar directamente en el trabajo etnográfico. Agradecer a los miembros del grupo MIRCo por facilitarle el acceso a estos datos, y la lectura de los diarios de campo. El proyecto al que se describe: "Escuela multilingüismo: un studio sociolingüístico critic de los programas de educación lingüística en la Comunidad de Madrid" HUM2007-64694/FILO. Se agradece a Miriam Jiménez y Guillermo De Eugenio por los comentarios y correcciones del presente manuscrito. 